\title{
Application of the perfectly matched layer in 3D marine controlled-source electromagnetic modelling
}

\author{
Gang $\mathrm{Li}^{1,2}$ and Bo $\mathrm{Han}^{1}$ \\ ${ }^{1}$ College of Marine Geosciences, Ocean University of China, Qingdao 266100, China \\ ${ }^{2}$ Department for Geodynamics, GEOMAR Helmholtz Centre for Ocean Research Kiel, Kiel 24148, Germany \\ E-mail: gli@geomar.de
}

\section{SUMMARY}

In this study, the complex frequency-shifted perfectly matched layer (CFS-PML) in stretching Cartesian coordinates, is successfully applied to three-dimensional (3D) frequency-domain marine controlled-source electromagnetic (CSEM) field modelling. The Dirichlet boundary, which is usually used within the traditional framework of EM modeling algorithms, assumes the electric or magnetic field values are zero at the boundaries. This requires the boundaries be sufficiently far away from the sources in the area of interest. To mitigate the boundary artifacts, a large modelling area may be necessary even though cell sizes are allowed to grow toward the boundaries due to the diffusion of the electromagnetic wave propagation. Compared with the conventional Dirichlet boundary, the PML boundary is preferred as the modelling area of interest could be restricted to the target region and only a few absorbing layers surrounding can effectively depress the artificial boundary effect without losing the numerical accuracy. Furthermore, for joint inversion of seismic and marine CSEM data, if we used the PML for CSEM field simulation instead of the conventional Dirichlet, the modeling area for these two different geophysical data collected from the same survey area could the same, which is convenient for joint inversion grid 
matching. We apply the CFS-PML boundary to 3D marine CSEM modelling by using the staggered finite-difference (SFD) discretization. Numerical test indicates that the modeling algorithm using the CFS-PML also shows good accuracy compared to the Dirichlet. Furthermore, the modeling algorithm using the CFS-PML shows advantages in computational time and memory saving than that using the Dirichlet boundary. For the 3D example in this study, the memory saving using the PML is nearly $42 \%$ and the time saving is around $48 \%$ compared to using the Dirichlet.

Key words: Marine electromagnetics; Controlled source electromagnetics (CSEM); Numerical modelling; Perfectly matched layer.

\section{INTRODUCTION}

Marine controlled-source electromagnetic (CSEM) method is widely used for investigating the hydrocarbon and gas hydrate resources (Constable 2010). For frequency-domain marine CSEM survey, a towed electric dipole is usually used to generate the EM fields and the recorded data from receivers located at the seafloor can be used to analyze the resistivity distribution of the seafloor. Nowadays three dimensional (3D) EM modelling studies are of increasing importance for interpreting the EM data acquired in complex geologic settings (Avdeev 2005; Zhdanov 2010).

In the 3D EM modelling problem, the finite-difference/finite-volume, finite-element and integral equation methods are usually used and these methods are well studied in former publications (e.g., Börner 2010; Kelbert et al. 2014). In this study, the staggered finite-difference (SFD) method is used for modeling the 3D marine CSEM fields, which can produce accurate and reliable numerical solutions with a fast computation rate (Mackie et al. 1993; Smith 1996; Weiss \& Constable 2006; Sasaki \& Meju 2009; Mittet 2010). For these traditional frameworks of 3D EM modeling algorithms, a Dirichlet boundary is usually used, which assumes the electric or magnetic field values are zero at the boundaries. This is a truncated boundary and it requires the boundaries be sufficiently far away from the area of interest. To mitigate the boundary artifacts, a large modelling area may be necessary though cell sizes are allowed to grow toward the boundaries due to the diffusion of the electromagnetic wave propagation. An alternative way is to use the perfectly matched layer (PML), which is the common choice for seismic wave propagating simulation (e.g., Hu et al. 2007; Pan et al. 2012). By using the 
PML, the modelling area of interest could be restricted to the target region and only a few surrounding absorbing layers can effectively depress the artificial boundary effect without losing the numerical accuracy. It is worth mentioning that, for joint inversion of marine CSEM and seismic data, if we used the PML for CSEM field simulation instead of the conventional Dirichlet, the modeling area for these two different geophysical data collected from the same survey area could be the same, which is convenient for joint inversion grid matching (Hu et al. 2009).

Chen et al. (1997) applied Bérenger's PML to two-dimensional (2D) transient EM modelling problem using the finite-difference time-domain (FDTD) modeling of lossless media (Bérenger 1994). Schwarzbach et al. (2011) successfully utilized Bérenger's PML in 3D frequencydomain CSEM diffusing modelling problem with adaptive higher order finite element method. Similarly, Mittet (2010), de la Kethulle de Ryhove \& Mittet (2014) used Bérenger's PML in 3D marine magnetotelluric modelling by a finite-difference time-domain algorithm in which Maxwell's equations are solved in a fictitious-wave domain. The original PML proposed by Bérenger (1994) can be optimized and simply implemented in the form of the complex coordinate stretching along Cartesian coordinates (Chew \& Weedon 1994; Chew \& Jin 1996). To deduce the asymptotic behaviors at low and high frequencies, a complex frequency-shifted PML (CFS-PML) is also developed which greatly improve the absorbing performance of PML (Kuzuoglu \& Mittra 1996; Bérenger 2002). Hu et al. (2017) applied the CFS-PML boundary condition for transient electromagnetic modelling using a fictitious wave domain method. In this study, we present a novel 3D frequency-domain marine CSEM modeling algorithm, in which CFS-PML is successfully applied.

This study can be divided into three parts. We first give an introduction of 3D frequencydomain CSEM modeling scheme using the SFD grids with the CSF-PML. To avoid the source singularities, the secondary-field approach is used and the primary fields excited by the electric dipole source could be calculated quasi-analytically for the one-dimensional (1D) layered background. Then we compare the performance of CFS-PML with the conventional Dirichlet boundary by numerical analysis. Finally we give the conclusion that the CFS-PML modelling scheme proposed shows shows advantages in computational time and memory saving compared to using the Dirichlet boundary without losing numerical accuracy. 


\section{FORMULATIONS}

\section{$2.1 \quad$ Governing equations}

The electric/magnetic fields can be split into a primary part and a secondary (or scattered) part to avoid source-point singularities (Newman \& Alumbaugh 1995; Streich 2009; Sasaki \& Meju 2009). The primary fields are excited by the arbitrarily oriented dipole sources and can be computed quasi-analytically following Li \& Li (2016). The secondary fields are computed by the frequency-domain SFD method (Yee 1966).

Assuming that the time factor is $e^{-\mathrm{i} \omega t}$ where $\mathrm{i}^{2}=-1$, by using the electric dipole source, the Maxwell's equations in the frequency domain can be written as

$$
\nabla \times \mathbf{E}^{S}-\mathrm{i} \omega \mu_{0} \mathbf{H}^{S}=0, \quad \nabla \times \mathbf{H}^{S}-\sigma^{*} \mathbf{E}^{S}=\left(\sigma^{*}-\sigma^{P *}\right) \mathbf{E}^{P},
$$

where superscripts $P$ and $S$ are for the primary and secondary fields, respectively. $\mathbf{E}$ and $\mathbf{H}$ are the electric and magnetic fields, $\omega$ is the angular frequency, $\mu_{0}$ is the magnetic permeability in free space, and the complex conductivity $\sigma^{*}=\sigma-i \omega \varepsilon_{0}$ consists of the electric conductivity $\sigma$ and permittivity $\varepsilon_{0}$ in free space. The secondary-source term is given by $\left(\sigma^{*}-\sigma^{P *}\right) \mathbf{E}^{P}$ where $\sigma^{*}=\sigma^{P *}+\sigma^{S *}$.

After eliminating $\mathbf{H}^{S}$, eq. (1) becomes

$$
\nabla \times \nabla \times \mathbf{E}^{S}-\mathrm{i} \omega \mu_{0} \sigma^{*} \mathbf{E}^{S}=\mathrm{i} \omega \mu_{0}\left(\sigma^{*}-\sigma^{P *}\right) \mathbf{E}^{P} .
$$

In this study, we use eq. (2) for simulating marine CSEM fields with the frequency range of 0.1 to $10 \mathrm{~Hz}$.

\subsection{Implementation of CFS-PML}

In the complex stretched coordinate approach (Chew \& Weedon 1994; Kuzuoglu \& Mittra 1996), the governing equation using CFS-PML can be rewritten as

$$
\nabla_{h} \times \nabla_{e} \times \mathbf{E}^{S}-\mathrm{i} \omega \mu_{0} \sigma^{*} \mathbf{E}^{S}=\mathrm{i} \omega \mu_{0}\left(\sigma^{*}-\sigma^{P *}\right) \mathbf{E}^{P} .
$$

where $\nabla_{e}=\left(\frac{1}{\gamma_{x}^{e}} \partial_{x}, \frac{1}{\gamma_{y}^{e}} \partial_{y}, \frac{1}{\gamma_{z}^{e}} \partial_{z}\right), \nabla_{h}=\left(\frac{1}{\gamma_{x}^{h}} \partial_{x}, \frac{1}{\gamma_{y}^{h}} \partial_{y}, \frac{1}{\gamma_{z}^{h}} \partial_{z}\right), \gamma_{\nu}^{e}$ and $\gamma_{\nu}^{h}(\nu=x, y, z)$ are the complex stretched coordinate factors (decay factors) for electric and magnetic field components, respectively. SFD discretization of eq. (3) are similar as Newman \& Alumbaugh (1995) and Streich et al. (2013) (Appendix A). The entire computational domain is divided into $N_{\text {cell }}=N_{x} \times N_{y} \times N_{z}$ uniform cells, where $N_{x}, N_{y}$, and $N_{z}$ are the number of cells in the $x-, y$-, and $z$ - direction, respectively. After discretization, eq. (3) can be written in the matrix form as 
where $\mathbf{K}$ is a symmetric complex matrix of dimension $\left(3 N_{x} \times N_{y} \times N_{z}\right)^{2}$; the unknown vector $\mathrm{U}$ of length $3 N_{x} \times N_{y} \times N_{z}$ contains the electric field values $E_{x}^{S}, E_{y}^{S}$ and $E_{z}^{S}$ for all nodes; and $\mathbf{P}$ of length $3 N_{x} \times N_{y} \times N_{z}$ is the secondary source vector of the right-hand side of eq. (4) given by eq. (3). The entries in $\mathbf{K}$ depend on the grid spacing and the frequency-dependent medium properties, and $\mathbf{K}$ is up to 13 nonzero entries per line. The $3 \mathrm{D}$ array electric field values $E_{i+\frac{1}{2}, j, k}^{x S}, E_{i, j+\frac{1}{2}, k}^{y S}$, and $E_{i, j, k+\frac{1}{2}}^{z S}$, where the subscripts $i=1, \ldots, N_{x}, j=1, \ldots, N_{y}$, and $k=1, \ldots, N_{z}$, can be mapped into a $1 \mathrm{D}$ column array of indices $(i+1 / 2, j, k) \rightarrow[(i+$ $\left.1 / 2-1) \times N_{y}+j\right] \times N_{z}+k,(i, j+1 / 2, k) \rightarrow\left[(i-1) \times N_{y}+j+1 / 2\right] \times N_{z}+k$, and $(i, j, k+1 / 2) \rightarrow\left[(i-1) \times N_{y}+j\right] \times N_{z}+k+1 / 2$, respectively. Similarly, we can write the source term in a $1 \mathrm{D}$ column array. The stiffness matrix $\mathbf{K}$ is symmetric but highly sparse. Fig. 1 shows an example of the symmetric structure of the stiffness matrix $\mathbf{K}$ where a $5 \times 5 \times 5$ staggered grid is used.

The formed linear equations in (4) given by the discretization of eq. (3) are solved by a multifrontal direct solver MUMPS 5.0.2 parallelized by OpenMP (Amestoy et al. 2001, 2012), which could avoid uncertainties in pre-conditioning and convergence for iterative solutions, especially for low frequencies (Farquharson \& Miensopust 2011; Oldenburg et al. 2013). In this section, we will focus on the implementation of CFS-PML in details.

Following Chew \& Weedon (1994) and Bérenger (2007), the PML decay factors are given as

$$
\begin{aligned}
& \gamma_{\nu}^{e}=1-\mathrm{i} \eta_{\nu}^{e} / \omega, \quad \nu=x, y, z \\
& \gamma_{\nu}^{h}=1-\mathrm{i} \eta_{\nu}^{h} / \omega, \quad \nu=x, y, z
\end{aligned}
$$

Absorbing boundaries at the edges of the simulation region may be created by choosing appropriate values of $\eta_{\nu}$. Usually, the decay factor $\eta_{\nu}$ varies gradually from 0 at the PML/nonPML interface to its maximum at the outer boundaries of the computational domain to minimize the numerical reflections caused by spatial discretization (Hu et al. 2007).

In this study, the PML decay factor $\eta_{\nu}$ is determined empirically using the polynomial form of the PML (Hu et al. 2007). For example, the artificial attenuation along the $x$-direction is defined as (Bérenger 1996; Pan et al. 2012):

$$
\eta_{x}=\left\{\begin{array}{cl}
\eta_{\max }\left(-\frac{x}{L_{\mathrm{pml}}}\right)^{m}, & \text { if }-L_{\mathrm{pml}} \leq x<0 \\
0, & \text { if } 0 \leq x \leq 1 \\
\eta_{\max }\left(\frac{x-1}{L_{\mathrm{pml}}}\right)^{m}, & \text { if } 1<x \leq 1+L_{\mathrm{pml}}
\end{array}\right.
$$




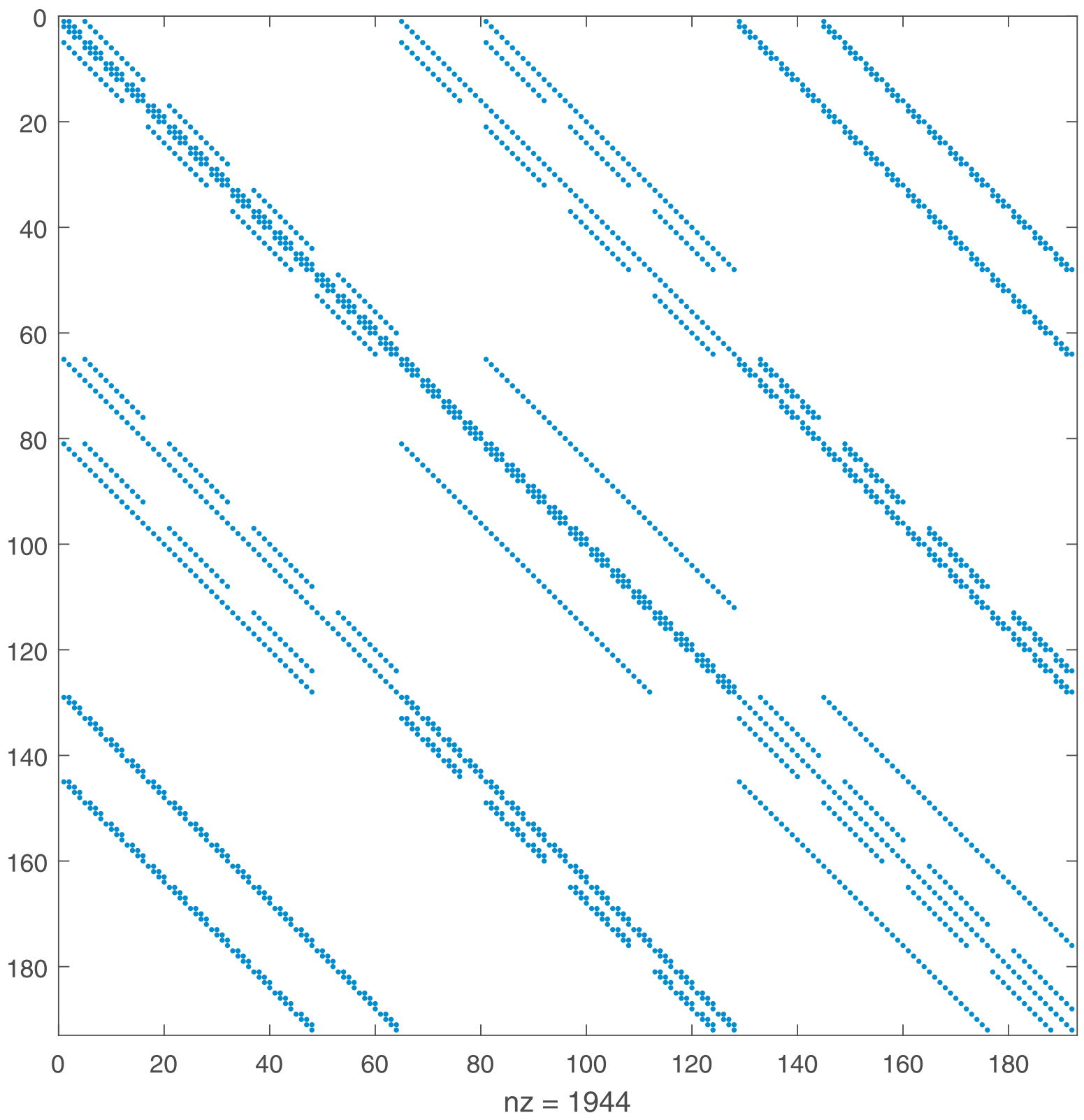

Figure 1. The schematic map showing different PML layers surrounding the 3D original computational cube. The left plot shows the original computational volume, which is a cuboid without PML layers. The right plot shows the PML layers surrounding the surrounding the original computational volume. The PML layers can be divided into 7 different types, which have different values of $\eta_{x}, \eta_{y}$ and $\eta_{z}$ (see Table 1).

where $\mathrm{m}$ is either 2 or 3 , and this value depends on the size of the computational domain. As pointed out by Bérenger (2002), $\mathrm{m}=3$ has a better absorption effect on the evanescent region than $\mathrm{m}=2$, whereas $\mathrm{m}=2$ has a better absorption effect on the traveling region. We used $\mathrm{m}=2$ in this study. The length of the interior domain is scaled to $1, L_{\mathrm{pml}}$ is the 


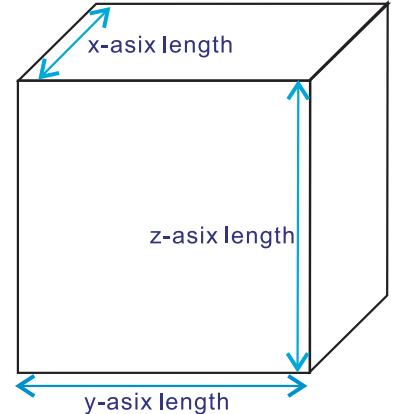

Original computational area
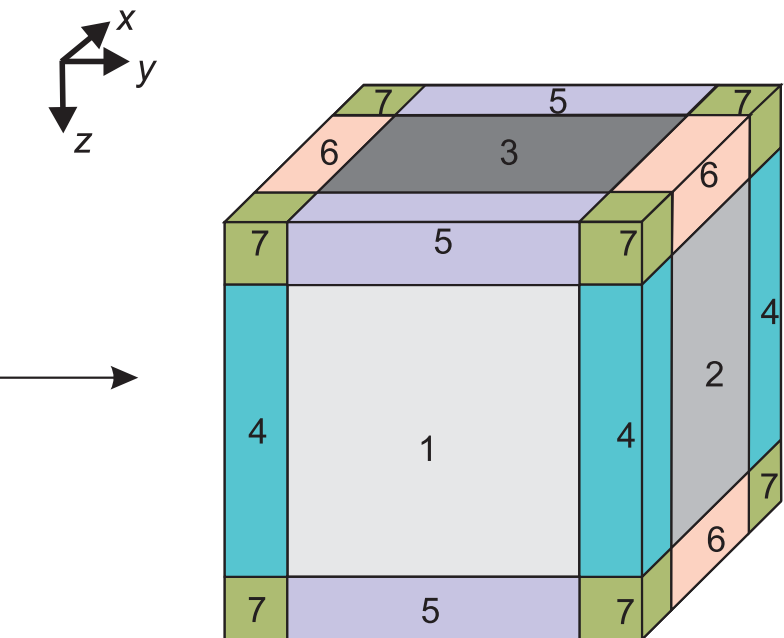

1 PML-X

2 PML-y

3 PML-Z

4 PML- $x, y$

5 PML-y,z

6 PML-Z,X

7 PML-x,y,z

Figure 2. Illustration of the symmetric structure of the stiffness matrix for a $5 \times 5 \times 5$ staggered grid. Note that only the locations of nonzero entries before applying the Dirichlet or PML boundary condition are shown (blue points). $n z=1944$ is the number of nonzero entries of the stiffness matrix.

thickness of the PML layer on each side, $\eta_{\max }$ is the maximum PML decay factor, and $m$ is an integer (e.g., two or three). We use the same definitions for the artificial attenuation parameters in the $y$ - and $z$-directions. Fig. 2 shows the different PML layers surrounding the original computational area. Note for the computation of the solution inside the PML layers in the $x$-direction, $\eta_{x}$ is positive and $\eta_{y}, \eta_{z}=0$. For PML layers in the $y$-direction, $\eta_{y}$ is positive and $\eta_{x}, \eta_{z}=0$. For PML layers in the $z$-direction, $\eta_{z}$ is positive and both $\eta_{x}, \eta_{y}=0$ (see Table 1). For PML layers in the corners, where we need damping in all directions, $\eta_{x}, \eta_{y}$ and $\eta_{z}$ are all positive (Collino \& Tsogka 2001).

The complex frequency stretched (CFS) PML, as introduced in Kuzuoglu \& Mittra (1996), Roden \& Gedney (2000) and Pan et al. (2012), is one of a number of approaches that are suitable to mimic the reflection-free boundaries at the outermost surface of the interior domain.

Table 1. The damping factors $\eta_{\nu}(\nu=x, y, z)$ for different PML layers.

\begin{tabular}{cccccccc}
\hline PML layers & PML-x & PML-y & PML-z & PML-x,y & PML-y,z & PML-z,x & PML-x,y,z \\
\hline$\eta_{x}$ & $>0$ & $=0$ & $=0$ & $>0$ & $=0$ & $>0$ & $>0$ \\
\hline$\eta_{y}$ & $=0$ & $>0$ & $=0$ & $>0$ & $>0$ & $=0$ & $>0$ \\
\hline$\eta_{z}$ & $=0$ & $=0$ & $>0$ & $=0$ & $>0$ & $>0$ & $>0$ \\
\hline
\end{tabular}


The complex frequency stretched coordinate factors are given by

$$
\begin{aligned}
& \gamma_{\nu}^{e}=\kappa_{\nu}^{e}+\frac{\eta_{\nu}^{e}}{\alpha_{\nu}^{e}+\mathrm{i} \omega}, \\
& \gamma_{\nu}^{h}=\kappa_{\nu}^{h}+\frac{\eta_{\nu}^{h}}{\alpha_{\nu}^{h}+\mathrm{i} \omega},
\end{aligned}
$$

where $\eta_{\nu}$ and $\kappa_{\nu}$ are positive real and $\kappa_{\nu}$ is $\geq 1$ (Bérenger 2007). $\eta_{\nu}$ are the PML decay factors, $\alpha_{\nu}$ are point-wise coefficients related to the CFS-PML (Roden \& Gedney 2000). The essence of the real parameter $\alpha_{\nu}$ is to absorb the evanescent waves and to improve the absorption performance at grazing angles (Bérenger 2002; Komatitsch \& Martin 2007; Martin et al. 2009). In this study, the parameter $\kappa_{\nu}$ is set as 1 , since the effect of these parameters for the absorption is not significant (Bérenger 2002).

In the PML region, the conductivity is equal to the value of the outmost cell of the interior domain. This strategy is employed to minimize the numerical reflection error at the interface between the PML domain and the interior domain.

We use similar setting for $\alpha_{\nu}$ as Roden \& Gedney (2000). We choose to make $\alpha_{\nu}$ vary in a linear fashion in their respective PML layer between a maximum value $\alpha_{\max }$ at the beginning (i.e., the entrance) of the PML and zero at its top. The maximum value of $\alpha_{\nu}$ is set as 95 percent of the transmitting frequency. With these settings, the discretized eq. (3) has the same formulas everywhere in space. The only difference between the PML domain and the interior domain equation is the value of the artificial attenuation $\eta_{\nu}$.

To reduce the discretization error, the parameter $\eta_{\nu}$ are scaled such that they are are 0 and 1 at the PML/interior volume interface, respectively, and are maximum at the exterior boundary. We use an optimal maximum attenuation $\left(\eta_{\max }\right)$ formulation similar as Komatitsch \& Martin (2007), Martin et al. (2009) and Pan et al. (2012)

$$
\eta_{\max }(x, y, z)=c \frac{(m+1) \sigma(x, y, z)}{L_{\mathrm{pml}}},
$$

where $\sigma(x, y, z)$ is the conductivity of the cell adjacent to the PML grid, $L_{\mathrm{pml}}$ is the thickness of the PML domain and $c$ a constant that determines the reflection from the PML interface. In this study, the empirical parameter $c$ is chosen to be several times of the skin depth for the background model.

\section{NUMERICAL ANALYSIS}

In this section, we examine the performance of the CFS-PML proposed. Two examples are tested, i.e., the 1D case compared with the quasi-analytical solutions given by Li \& Li (2016) and the 3D case compared with the sulutions of adaptive finite-element method (adaptive 
FEM) solutions given by MARE3DEM code (Zhang 2017). We have written a Fortran 90 code to implement the 3D SFD modeling scheme for marine CSEM data. The numerical accuracy, time consumption and memory saving are compared between the CFS-PML and the conventional Dirichlet boundary (Li et al. 2017). The numerical test is performed under the Dell Precision Tower 3620 (3.50 GHz CPU Intel@Xeon E3-1240 v5 family including 2 processors with up to 4 cores per processor, memory up to $16 \mathrm{G}$ ), which is suitable for OpenMP parallel programming. Note the MUMPS 5.0.2 used for factorizing the complex sparse matrix formed by SFD discretization is parallelized by OpenMP in an "out-of-core" environment (Amestoy et al. 2001, 2012). When the "out-of-core" phase is activated and the complete matrix of factors is written to disk and will be read each time a solution phase is requested, therefore the memory requirement can be significantly reduced while not increasing much of the factorization time on a reasonably small number of processors.

\subsection{The 1D case}

For simplicity, we first use a one-dimensional (1D) canonical reservoir model given by Constable \& Weiss (2006), so that our SFD numerical solutions can be easily validated by the results obtained from the quasi-analytical 1D algorithm ( $\mathrm{Li} \& \mathrm{Li} 2016$ ). The 1D canonical reservoir model consists of a seawater layer with $0.3 \Omega \mathrm{m}$ resistivity and $1 \mathrm{~km}$ thickness, a sediment layer with $1 \Omega m$ resistivity and a $1000 \mathrm{~m}$ thickness (see Fig. 3). The canonical reservoir is buried at a depth of $2 \mathrm{~km}$ below the sea surface with $100 \Omega \mathrm{m}$ resistivity and a $100 \mathrm{~m}$ thickness. Inline (transmitter pointing along $y$ - direction) transmissions are excited at a frequency of $0.25 \mathrm{~Hz}$. The transmitter location is $x=0, y=-5000, z=950 \mathrm{~m}$, and 51 receivers are located at the seafloor with $200-\mathrm{m}$ intervals from $y=-5000$ to $5000 \mathrm{~m}$ along the towed line. Here, we only consider the electric and magnetic fields in the transmitter-receiver plane at $x=0$.

The computational volume is $\{(x, y, z):-50 \mathrm{~km} \leq x, y, z \leq 50 \mathrm{~km}\}$ and it is divided into $76 \times 76 \times 72$ staggered-grids when using the Dirichlet boundary condition (Fig. 4a and $4 \mathrm{~b})$. The $x$ - and $y$ - grid spacing is the same and becomes larger towards the boundaries, and the $z$-grid spacing becomes larger with depth. The minimum horizontal spacing is set to be $200 \mathrm{~m}$, while the minimum vertical grid spacing is given as $50 \mathrm{~m}$. For the CFS-PML, the computational volume is $\{(x, y, z):-6.6 \mathrm{~km} \leq x, y \leq 6.6 \mathrm{~km},-8 \mathrm{~km} \leq z \leq 3 \mathrm{~km}\}$ and a $66 \times 66 \times 64$ staggered-grid including 8 PML layers surrounding the computational area is used (Fig. 4c).

Fig. 5 shows the 3D SFD solutions compared to 1D quasi-analytic solutions for the frequency $0.25 \mathrm{~Hz}$. From Fig. 5 we can see that, the numerical accuracy is acceptable for both 


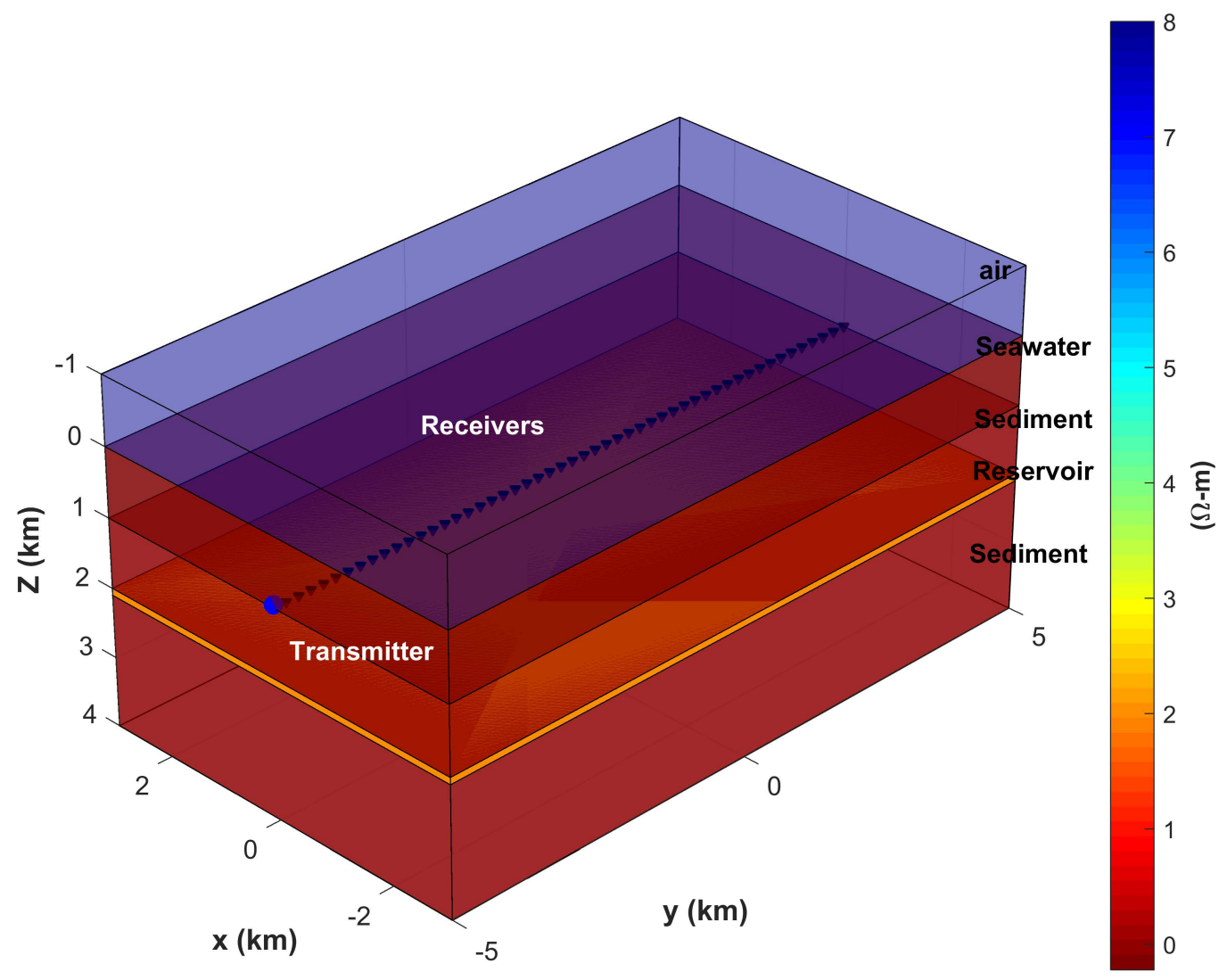

Figure 3. An 1D canonical reservoir model similar to Constable \& Weiss (2006). There are five layers including air layer, seawater layer and three seafloor layers. The $\bullet$ and $\mathbf{\nabla}$ indicate the transmitter and receivers, respectively.

the gridding using Dirichlet and CFS-PML boundary. The absolute errors of the amplitude for $E_{y}, E_{z}$ and $H_{x}$ are no more than $2 \%, 4 \%$ and $2 \%$, respectively. The relative errors of the phase for $E_{y}, E_{z}$ and $H_{x}$ are all less than $1^{\circ}$. When using the CFS-PML boundary, less grids are used so that both the memory and computation time saving are significant (Table 2). For this $1 \mathrm{D}$ test, the memory saving is nearly $43 \%$ and the time saving is around $50 \%$.

Similar as Roden \& Gedney (2000) and Hu et al. (2017), the reflection errors at the receiver positions are calculated using

$$
\text { Reflection error }=20 \log _{10}\left|\frac{\operatorname{abs}\left(\mathbf{F}_{i}\right)-\operatorname{abs}\left(\mathbf{F}_{i}^{0}\right)}{\operatorname{abs}\left(\mathbf{F}_{i}^{0}\right)}\right|, i=1, \ldots, N_{R_{x}}
$$

where $N_{R_{x}}$ is the number of receivers, $\mathbf{F}$ represents the electric $\mathbf{E}$ or magnetic field $\mathbf{H}$ observed at the receiver locations using the CFS-PML boundary, $\mathbf{F}^{0}$ represents the reference field with 

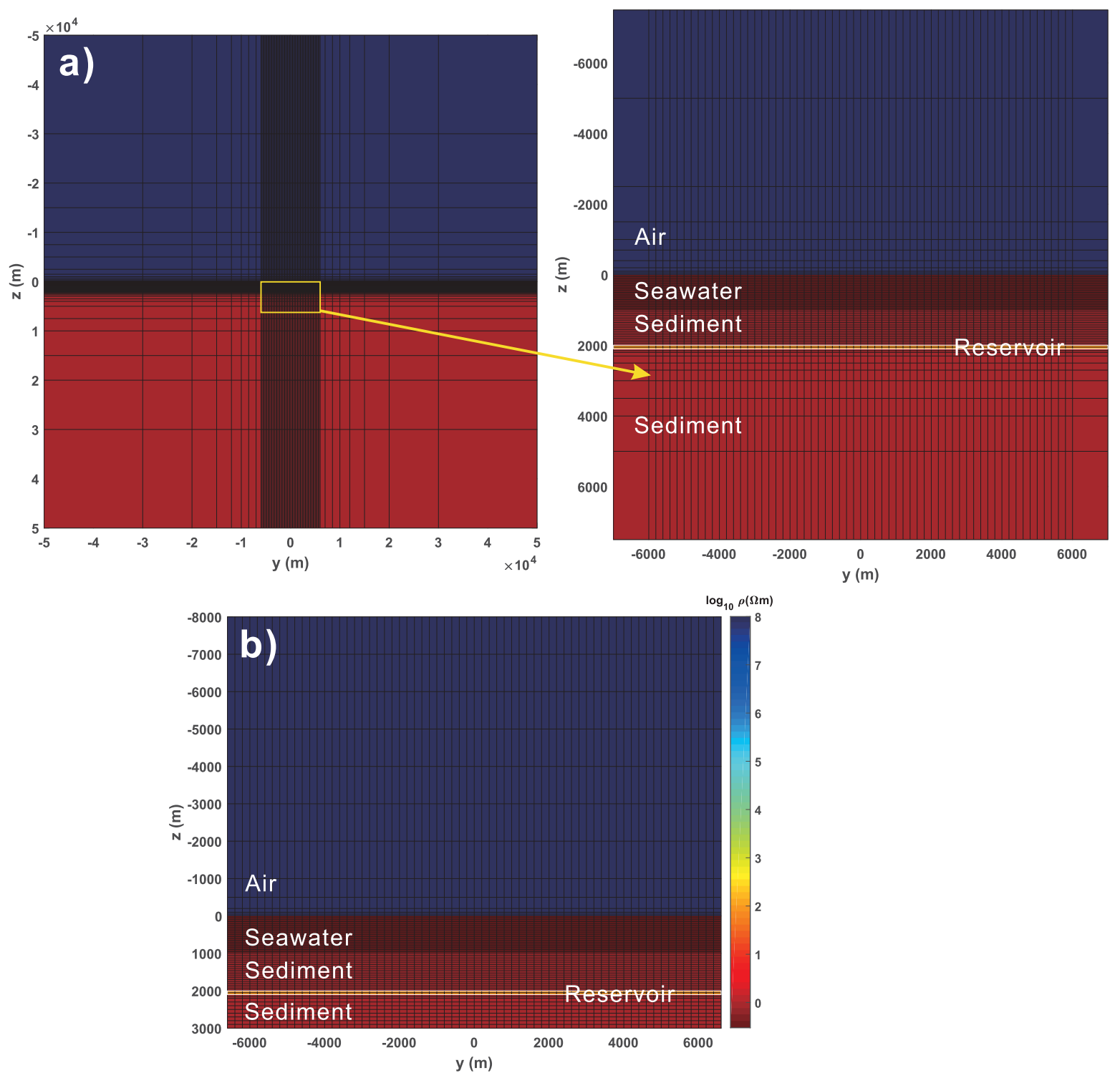

Figure 4. A $2 \mathrm{D}$ cutaway of the meshin the transmitter-receiver plane at $x=0$ used for forward calculation of the 1D canonical reservoir model in Fig. 3 using a 3D SFD code with a PML and Dirichlet boundary condition. a) The mesh using the conventional Dirichlet boundary, in which local mesh for reservoir area bounded by yellow box is enlarged. b) Local mesh using the CFS-PML boundary.

boundaries distant enough to avoid any boundary reflections that might interfere with the observed data. In this example, the 1D quasi-analytical results are taken as the reference fields.

Fig. 6 shows the reflection error for both the electric field (Fig. 6a) and the magnetic field (Fig. 6b) at the receiver positions for the $1 \mathrm{D}$ model shown in Fig. 3. Actually, the reflection errors at the receiver positions in eq. (11) indicate the total electric or magnetic field reflection level. The reflection error of both $\mathbf{E}$ and $\mathbf{H}$ using CFS-PML boundary is low 
a)

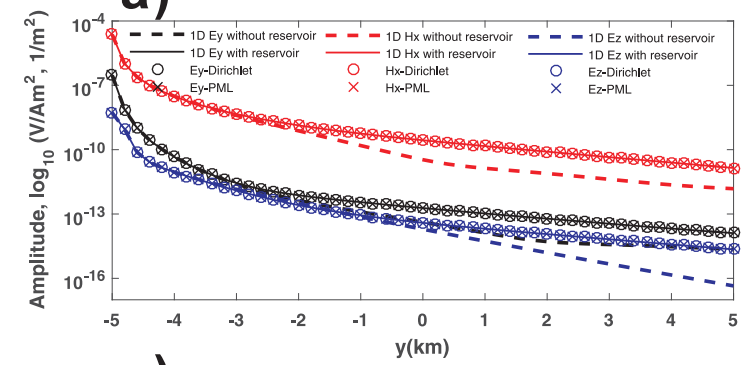

c)

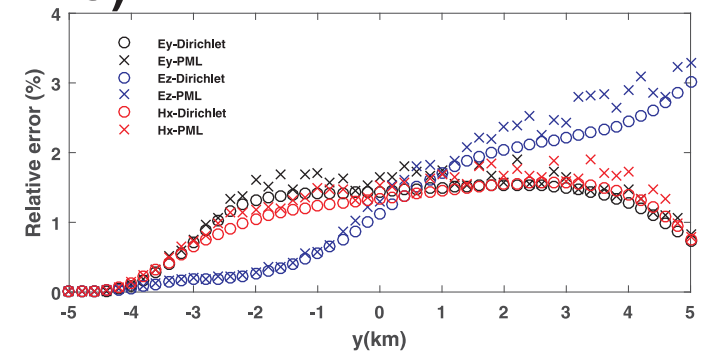

b)

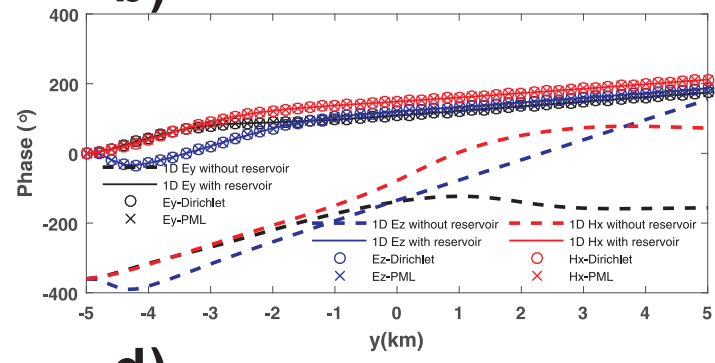

d)

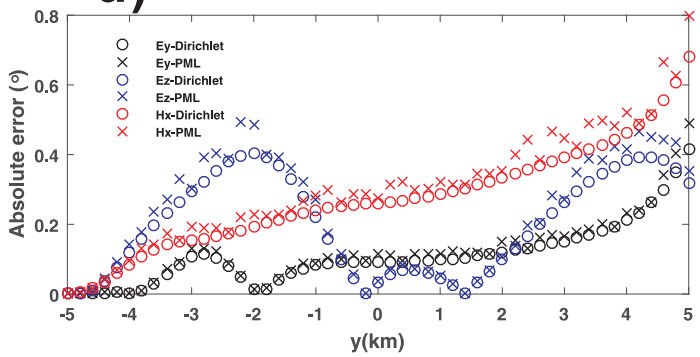

Figure 5. 3D numerical solutions against 1D quasi-analytic solutions for the frequency $0.25 \mathrm{~Hz}$. The $\mathrm{y}$ axis in $\mathrm{km}$ is also the source receiver offset. The SFD solutions with the CFS-PML boundary are compared to those with the Dirichlet boundary condition given by Li et al. (2017). a) Amplitude of the electric field $\mathbf{E}$ and electric field $\mathbf{H}$, in which the units are $\mathrm{V} / \mathrm{Am}^{2}$ and $1 / \mathrm{m}^{2}$, respectively. b) Phase in degrees for the electric field $\mathbf{E}$ and electric field $\mathbf{H}$. c) Relative error of amplitude for the electric field $\mathbf{E}$ and electric field $\mathbf{H}$ compared to the 1D analytic solution given by Li \& Li (2016). d) Absolute error of phase for the electric field $\mathbf{E}$ and electric field $\mathbf{H}$ compared to the 1D analytic solution. The solid line indicates the quasi-analytic results for the 1D model in Fig. 3, $\times$ and $\bigcirc$ indicate the SFD results using the Dirichlet boundary and the PML boundary, respectively. The colors black, blue and red are for $E_{y}, E_{z}$ and $H_{x}$, respectively.

(less than -30 dB noise which corresponds to the relative error of $3 \%$ ), which is similar as that using the Dirichlet boundary. This indicates that the CFS-PML boundary effectively depress the artificial boundary effect.

Table 2. Comparison between the CFS-PML boundary and the conventional Dirichlet boundary for the $1 \mathrm{D}$ example.

\begin{tabular}{cccccc}
\hline Boundary & Gridding & PML layers & Number of unknowns & Memory used (G) & Total time used (min) \\
\hline Dirichlet & $76 \times 76 \times 72$ & - & 1247616 & $\approx 7.76$ & $\approx 14.15$ \\
\hline CFS-PML & $66 \times 66 \times 64$ & 8 & 836352 & $\approx 4.41$ & $\approx 7.04$ \\
\hline
\end{tabular}



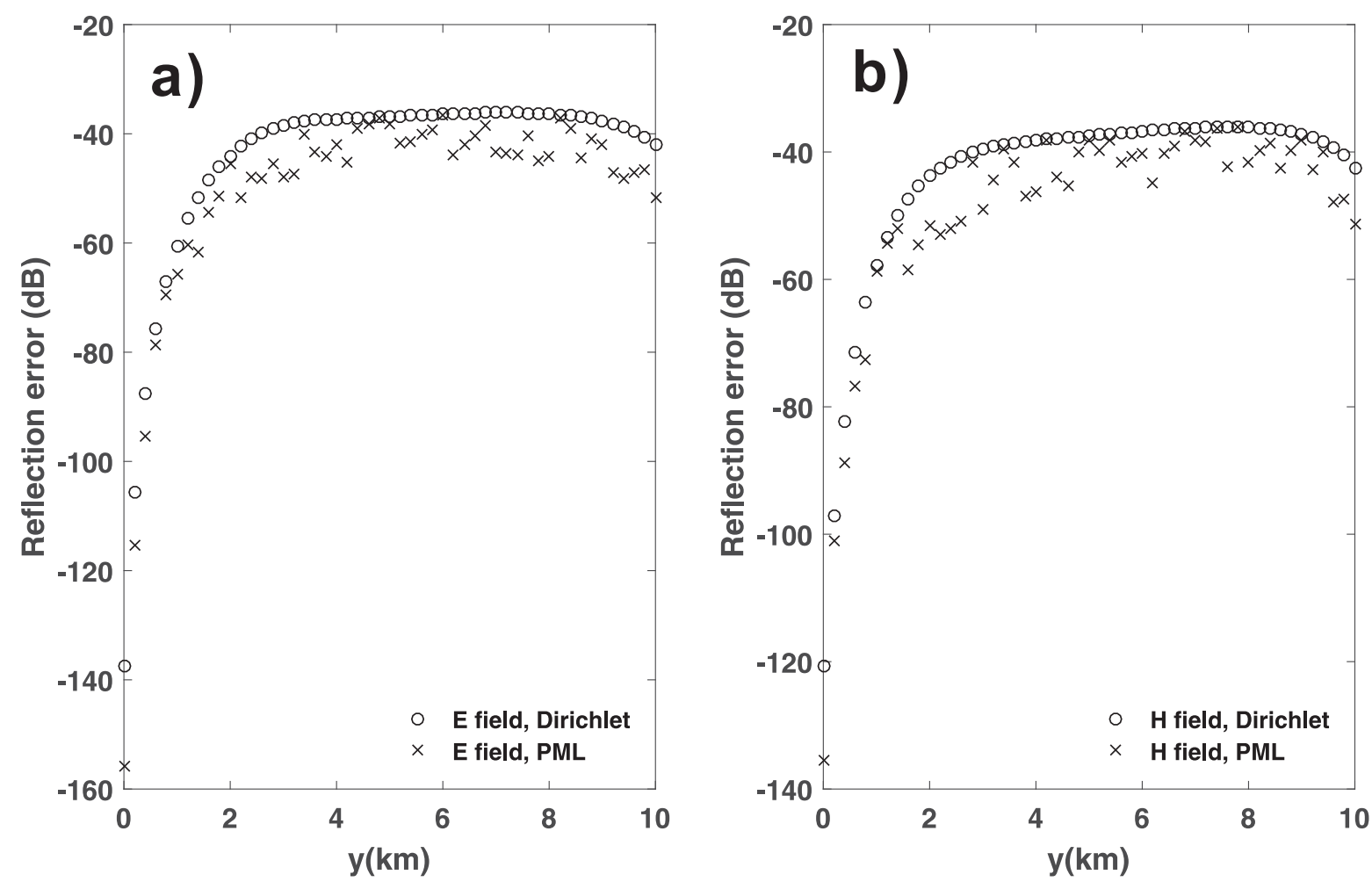

Figure 6. Reflection error of the PML and Dirichlet boundaries for the 1D model shown in Fig. 3. The symbols $\times$ and $\bigcirc$ are for the Dirichlet boundary and the PML boundary, respectively. a) Reflection error for the electric field $\mathbf{E}$, and b) for the magnetic field $\mathbf{H}$.

\subsection{The 3D case}

In this part, we present a 3D example similar as Weiss \& Constable (2006) (Fig. 7). The 3D geo-electric model consists of a reservoir represented by a $100-\mathrm{m}$ thick horizontal slab and resistivity $100 \Omega m$. The reservoir body is buried at a depth of $1 \mathrm{~km}$ within a half-space of $1 \Omega m$ water-saturated sediment. The thin slab is a cuboid with both $4 \mathrm{~km}$ length, $4 \mathrm{~km}$ width and $100 \mathrm{~m}$ height. The center of the top surface of the slab corresponds to the origin $o$ of the Cartesian coordinates used. Inline (transmitter pointing along $y$ - direction) transmissions are excited at a frequency of $1 \mathrm{~Hz}$. The transmitter location is $x, y=0, z=900 \mathrm{~m}$, and 21 receivers are located at the seafloor with $200-\mathrm{m}$ intervals from $y=0$ to $5000 \mathrm{~m}$ along the towed line. Only the electric and magnetic fields in the transmitter-receiver plane at $x=0$ are considered.

The computational volume is $\{(x, y, z):-50 \mathrm{~km} \leq x, y, z \leq 50 \mathrm{~km}\}$ is also divided into $78 \times 78 \times 74$ staggered-grids when using the Dirichlet boundary condition (Fig. 8a and 8b) For the CFS-PML, the computational volume is $\{(x, y, z):-6.6 \leq x, y \leq 6.6 \mathrm{~km},-8 \mathrm{~km} \leq z \leq$ $3 \mathrm{~km}\}$ and a $68 \times 68 \times 64$ staggered-grid including 8 PML layers surrounding the computational 


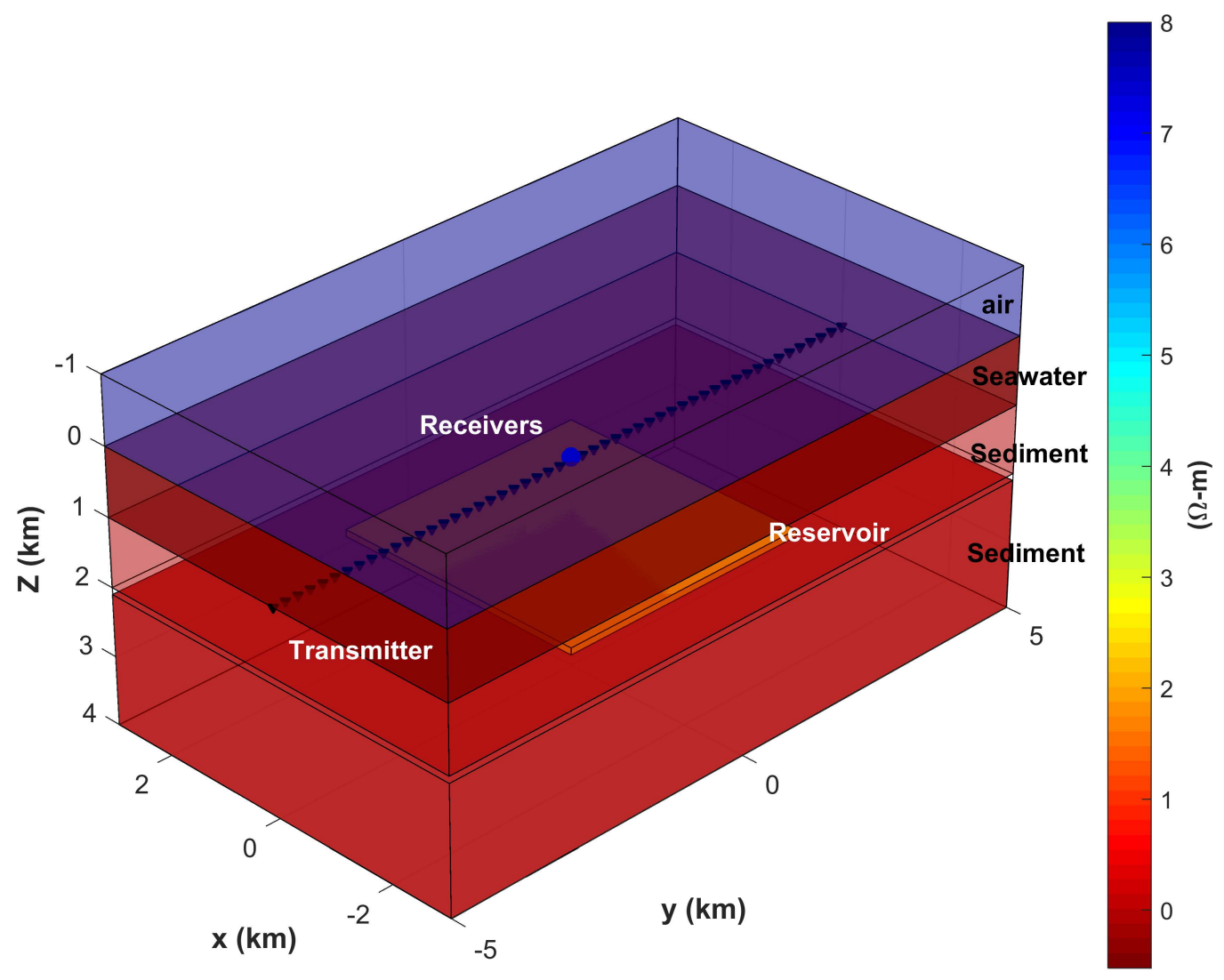

Figure 7. A 3D canonical reservoir model similar to Weiss \& Constable (2006). There are air layer, seawater layer and a canonical slab of the volume $\{(x, y, z):-2 \mathrm{~km} \leq x, y \leq 2 \mathrm{~km}, 2 \mathrm{~km} \leq z \leq 2.1 \mathrm{~km}\}$ which is embedded into a seafloor halfspace. The $\bullet$ and $\boldsymbol{\nabla}$ indicate the transmitter and receivers, respectively.

area is used (Fig. 8c). For comparison, the adaptive FEM solutions computed by MARE3DEM are used (Zhang 2017). The computational volume is $\{(x, y, z):-50 \leq x, y, z \leq 50 \mathrm{~km}\}$ and the total triangular elements are 755804 after 10 refinement iterations.

Fig. 9 shows the 3D SFD solutions compared to 3D adaptive FEM solutions for the frequency $0.25 \mathrm{~Hz}$. Fig. 9 shows a good numerical accuracy for using Dirichlet or CFS-PML boundary. The absolute errors of the amplitude for $E_{y}, E_{z}$ and $H_{x}$ are all no more than $1.2 \%$. The relative errors of the phase are all less than $1^{\circ}$. Both the memory and computation time saving are significant when using the CFS-PML boundary with less grids (Table 3). For the $3 \mathrm{D}$ test, the memory saving using the PML is nearly $42 \%$ and the time saving is around $48 \%$ 

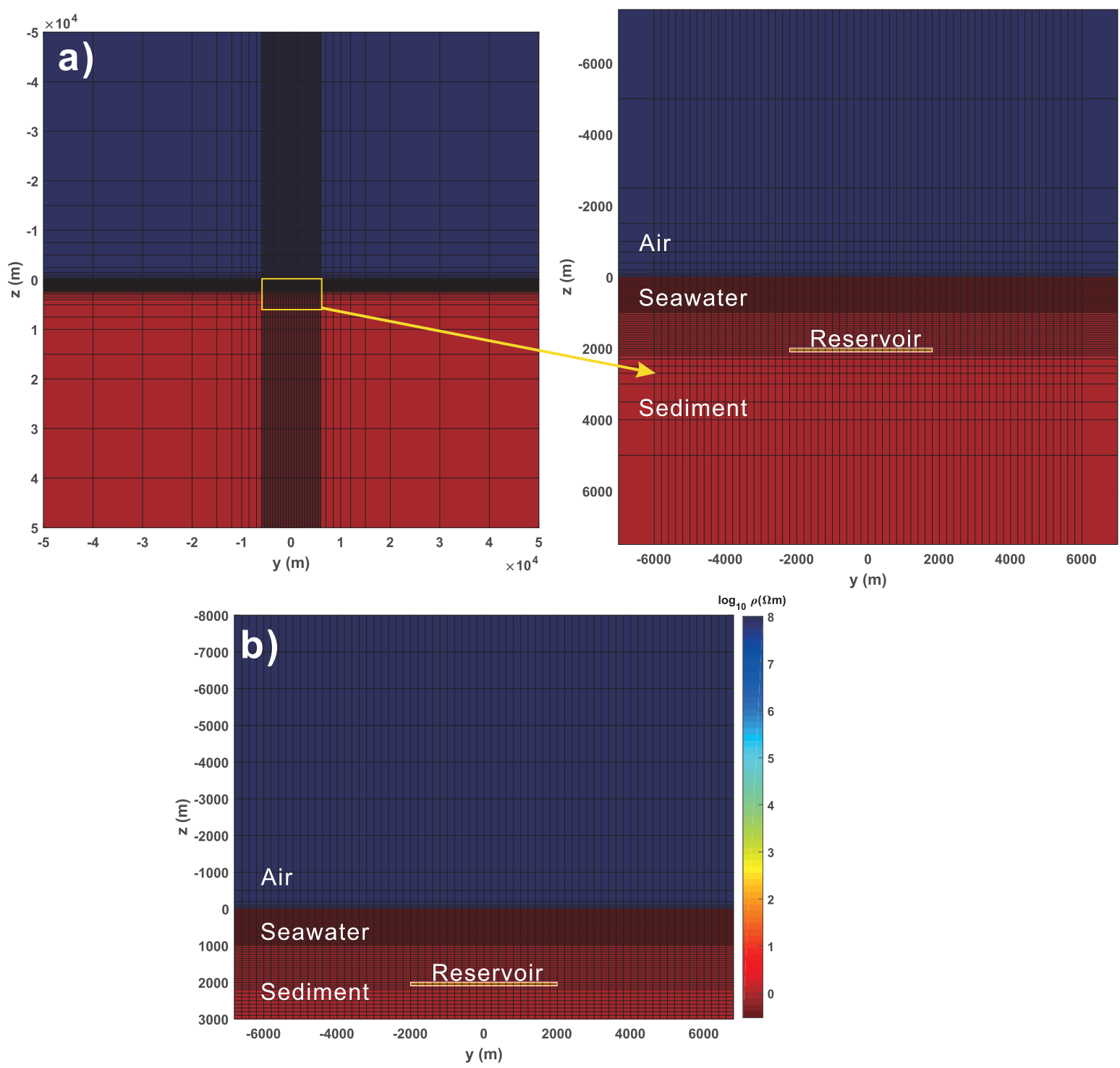

Figure 8. A 2D cutaway of the mesh in the transmitter-receiver plane at $x=0$ used for forward calculation of the 3D canonical reservoir model in Fig. 7 using a 3D SFD code with a PML and Dirichlet boundary condition. a) The mesh using the conventional Dirichlet boundary, in which local mesh for reservoir area bounded by yellow box is enlarged. b) Local mesh using the CFS-PML boundary.

compared to using the Dirichlet. In Fig. 9, one can distinguish the 3D adaptive finite-element results (solid line) and quasi-analytic results for the 1D background model without the 3D resistive slab (dashed line). This indicate the effect of the 3D thin slab is apparent.

We notice that the PML appears to have a little higher error compared to the Dirichlet for both the 1D and 3D examples. We think that because the modeling area is large enough and the boundaries are far away enough (the modeling area is set to be $\{(x, y, z):-50 \mathrm{~km} \leq$ $x, y, z \leq 50 \mathrm{~km}\})$, no or little boundary reflections appear for the Dirichlet. For the PML, 
a)
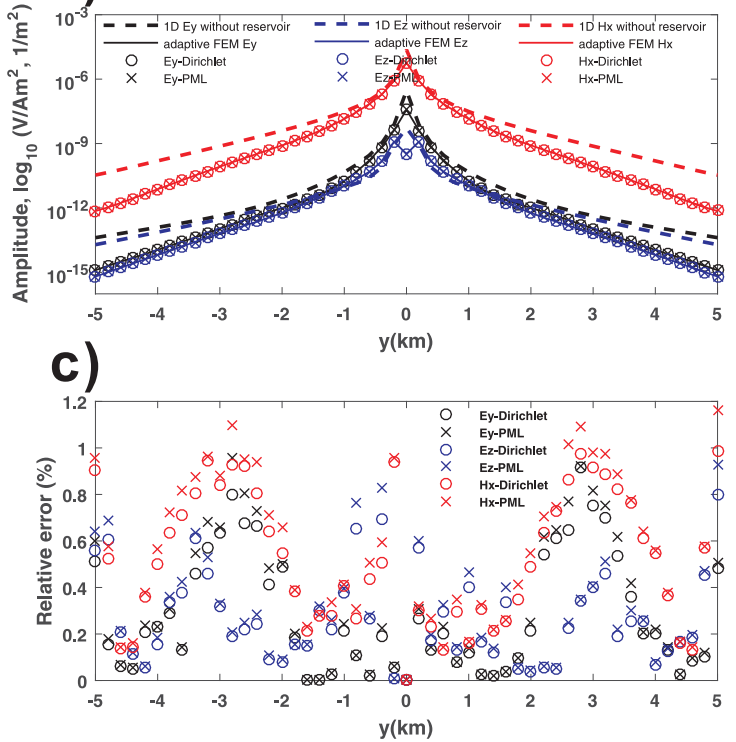

b)

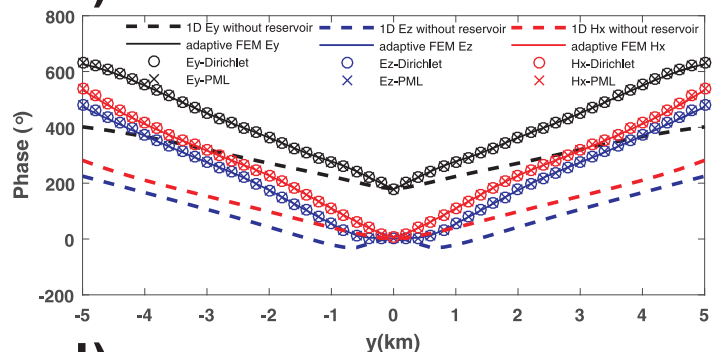

d)

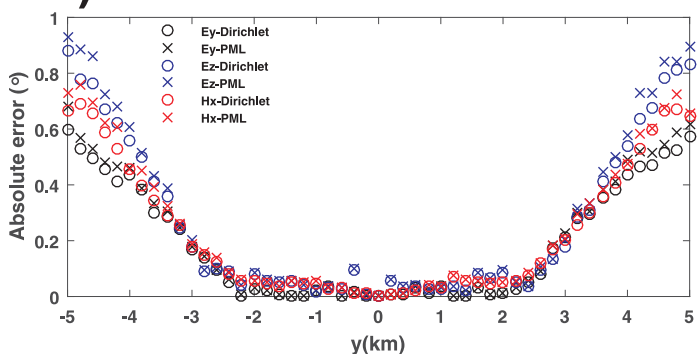

Figure 9. 3D numerical solutions against 3D adaptive FEM solutions of MARE3DEM given by Zhang (2017) for the frequency $1 \mathrm{~Hz}$. The SFD solutions with the CFS-PML boundary are compared to those with the Dirichlet boundary condition given by Li et al. (2017). Note that the thin slab is of dimension $-4 \mathrm{~km}$ to $4 \mathrm{~km}$ along $y$ axis. The solid line indicates the adaptive finite-element results, the dash line indicates the quasi-analytic results for the 1D background model in Fig. 7 without the 3D resistive slab, $x$ and $\bigcirc$ indicate the SFD results using the Dirichlet boundary and the PML boundary, respectively. The colors black, blue and red are for $E_{y}, E_{z}$ and $H_{x}$, respectively.

the modeling area for PML are still much smaller than that of Dirichlet. Although we use 10 PML layers which are proved to be sufficient enough for absorbing boundary reflections, the absorbing rate cannot reach 100 percent.

The reflection errors at the receiver positions for the 3D example are shown in Fig. 10. The 3D adaptive FEM results, using the Dirichlet boundary, are taken as the reference fields. In this test, the Dirichlet boundary for finite element simulation is far enough to avoid any boundary reflections that might interfere with the observed data. The reflection error of both $\mathbf{E}$ and $\mathbf{H}$ using CFS-PML boundary are less than $-30 \mathrm{~dB}$ and $-28 \mathrm{~dB}$, respectively, which are

Table 3. Comparison between the CFS-PML boundary and the conventional Dirichlet boundary for the $3 \mathrm{D}$ example.

\begin{tabular}{cccccc}
\hline Boundary & Gridding & PML layers & Number of unknowns & Memory used (G) & Total time used (min) \\
\hline Dirichlet & $78 \times 78 \times 74$ & - & 1350648 & $\approx 8.75$ & $\approx 15.46$ \\
\hline CFS-PML & $68 \times 68 \times 64$ & 8 & 887808 & $\approx 5.09$ & $\approx 8.08$ \\
\hline
\end{tabular}



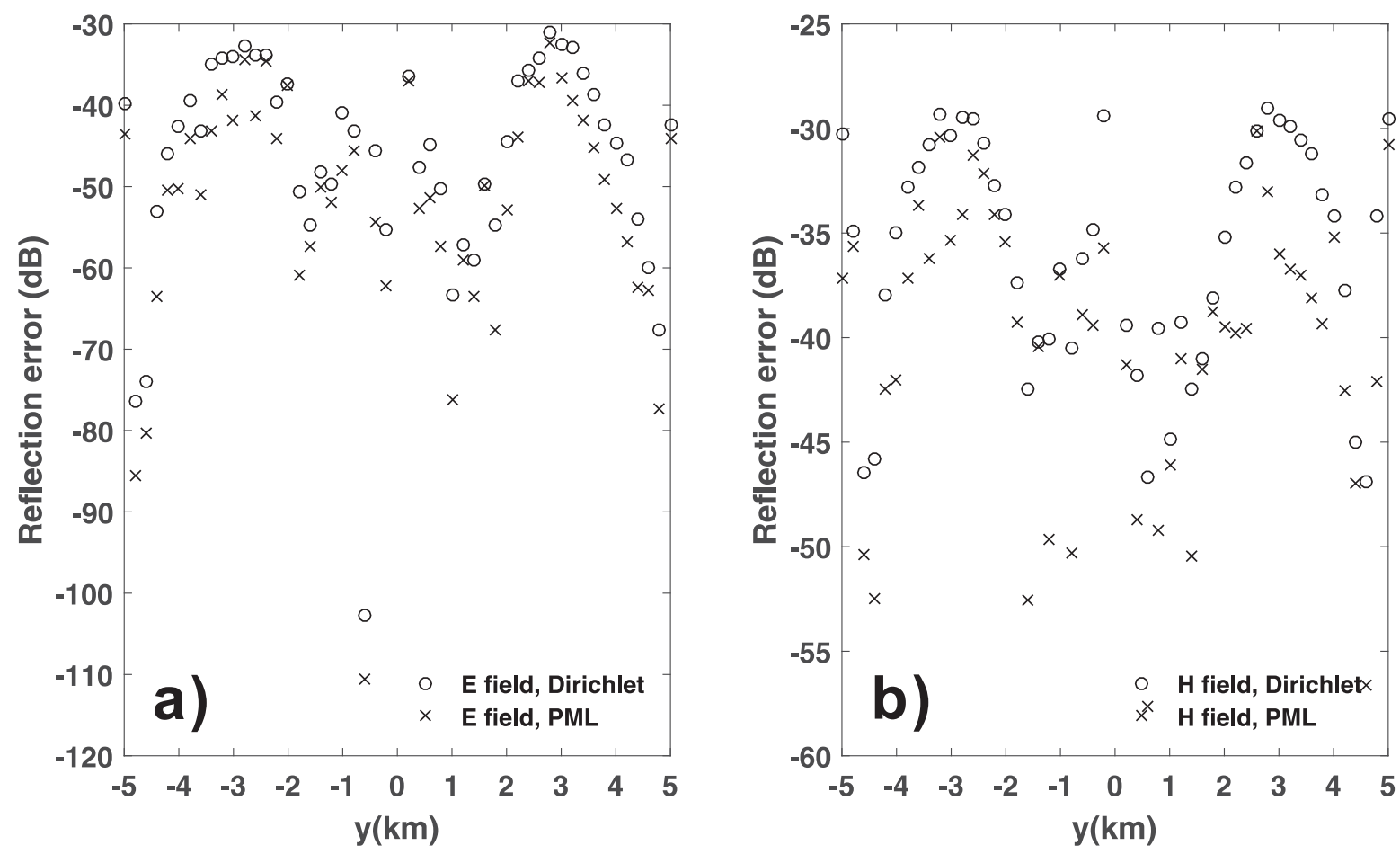

Figure 10. Reflection error of the PML and Dirichlet boundaries for the 3D model shown in Fig. 6 . Note that the thin slab is of dimension $-4 \mathrm{~km}$ to $4 \mathrm{~km}$ along $y$ axis. The symbols $\times$ and $\bigcirc$ are for the Dirichlet boundary and the PML boundary, respectively. a) Reflection error for the electric field $\mathbf{E}$, and b) for the magnetic field $\mathbf{H}$.

similar as those using the Dirichlet boundary. This indicates that the artificial boundary effect is effectively depressed by the CFS-PML boundary.

\section{CONCLUSIONS}

In this study, the complex frequency stretched perfectly matched layer (CFS-PML) has been applied to the 3D marine controlled-source electromagnetic (CSEM) forward problem. For the PML boundary, the model area can be restricted to the region of interest and only a few absorbing layers surrounding can effectively depress the artificial boundary effect without losing the numerical accuracy.

The next step is to develop a inversion scheme for interpreting real data based on the 3D marine CSEM modelling algorithm using CFS-PML. Furthermore, for developing a joint 3D inversion scheme of marine CSEM and seismic data, the proposed 3D CSEM modelling scheme using CFS-PML could be more convenient than using the conventional Dirichlet boundary condition. For seismic wave simulation, a PML boundary is commonly used. If we used the PML for CSEM field simulation, the modeling area for these two different geophysical data 
collected from the same survey area could be the same. This could avoid dealing with the grid matching when the CSEM gridding area using Dirichlet boundary is usually larger than the seismic gridding area (Hu et al. 2009).

\section{ACKNOWLEDGMENTS}

The authors acknowledge the editor Jörg Renner, the associate editor Mark Everett, and two anonymous reviewers for their constructive comments. Wenyi $\mathrm{Hu}$ is acknowledged for his fruitful discussions about the implementation of PML. Yuxiang Zhang is thanked for providing the $3 \mathrm{D}$ adaptive finite-element results using MARE3DEM. Gang Li thanks the support from the Sino-German (CSC-DAAD) Postdoc Scholarship Program 2016. This work is funded by the National Natural Science Foundation of China (41604063) and the Shandong Provincial Natural Science Foundation, China (ZR2016DQ15 and ZR2016DB31).

\section{REFERENCES}

Amestoy, P. R., Duff, I. S., L'Excellent, J.-Y., \& Koster, J., 2001. A fully asynchronous multifrontal solver using distributed dynamic scheduling, SIAM Journal on Matrix Analysis and Applications, 23(1), 15-41.

Amestoy, P. R., Duff, I. S., L’Excellent, J.-Y., Robert, Y., Rouet, F.-H., \& Uçar, B., 2012. On computing inverse entries of a sparse matrix in an out-of-core environment, SIAM Journal on Scientific Computing, 34(4), A1975-1999.

Avdeev, D. B., 2005. Three-dimensional electromagnetic modelling and inversion from theory to application, Surveys in Geophysics, 26(6), 767-799.

Bérenger, J.-P., 1994. A perfectly matched layer for the absorption of electromagnetic waves, Journal of Computational Physics, 114(2), 185-200.

Bérenger, J.-P., 1996. Three-dimensional perfectly matched layer for the absorption of electromagnetic waves, Journal of Computational Physics, 127(2), 363-379.

Bérenger, J.-P., 2002. Application of the CFS PML to the absorption of evanescent waves in waveguides, IEEE Microwave and Wireless Components Letters, 12(6), 218-220.

Bérenger, J.-P., 2007. On the huygens absorbing boundary conditions for electromagnetics, Journal of Computational Physics, 226(1), 354-378.

Bérenger, J.-P., 2007. Perfectly Matched Layer (PML) for Computational Electromagnetics, Morgan \& Claypool, 1st edn.

Börner, R.-U., 2010. Numerical modelling in geo-electromagnetics: Advances and challenges, Surveys in Geophysics, 31(2), 225-245. 
Chen, Y. H., Chew, W. C., \& Oristaglio, M. L., 1997. Application of perfectly matched layers to the transient modeling of subsurface EM problems, Geophysics, 62(6), 1730-1736.

Chew, W. C. \& Jin, J. M., 1996. Perfectly matched layers in the discretized space: An analysis and optimization, Electromagnetics, 16(4), 325-340.

Chew, W. C. \& Weedon, W. H., 1994. A 3D perfectly matched medium from modified Maxwell's equations with stretched coordinates, Microwave and Optical Technology Letters, 7(13), 599-604.

Collino, F. \& Tsogka, C., 2001. Application of the perfectly matched absorbing layer model to the linear elastodynamic problem in anisotropic heterogeneous media, Geophysics, 66(1), 294-307.

Constable, S., 2010. Ten years of marine CSEM for hydrocarbon exploration, Geophysics, 75(5), 75A67-75A 81 .

Constable, S. \& Weiss, C. J., 2006. Mapping thin resistors and hydrocarbons with marine EM methods: Insights from 1d modeling, Geophysics, 71(2), G43-G51.

de la Kethulle de Ryhove, S. \& Mittet, R., 2014. 3D marine magnetotelluric modeling and inversion with the finite-difference time-domain method, Geophysics, 79(6), E269-E286.

Farquharson, C. G. \& Miensopust, M. P., 2011. Three-dimensional finite-element modelling of magnetotelluric data with a divergence correction, Journal of Applied Geophysics, 75(4), 699-710.

Haber, E., Ascher, U., Aruliah, D., \& Oldenburg, D., 2000. Fast simulation of 3D electromagnetic problems using potentials, Journal of Computational Physics, 163(1), 150-171.

Hu, W., Abubakar, A., \& Habashy, T. M., 2007. Application of the nearly perfectly matched layer in acoustic wave modeling, Geophysics, 72(5), SM169-SM175.

Hu, W., Abubakar, A., \& Habashy, T. M., 2009. Joint electromagnetic and seismic inversion using structural constraints, Geophysics, 74(6), R99-R109.

Hu, Y., Egbert, G., Ji, Y., \& Fang, G., 2017. A novel CFS-PML boundary condition for transient electromagnetic simulation using a fictitious wave domain method, 52(1), 118-131.

Kelbert, A., Kuvshinov, A., Velimsky, J., Koyama, T., Ribaudo, J., Sun, J., Martinec, Z., \& Weiss, C. J., 2014. Global 3-D electromagnetic forward modelling: a benchmark study, Geophysical Journal International, 197(2), 785-814.

Komatitsch, D. \& Martin, R., 2007. An unsplit convolutional perfectly matched layer improved at grazing incidence for the seismic wave equation, Geophysics, 72(5), SM155-SM167.

Kuzuoglu, M. \& Mittra, R., 1996. Frequency dependence of the constitutive parameters of causal perfectly matched anisotropic absorbers, IEEE Microwave and Guided Wave Letters, 6(12), 447449 .

Li, G., Zhang, L., \& Han, B., 2016. Stable electromagnetic modeling using a multigrid solver on stretching grids: The magnetotelluric example, IEEE Geoscience and Remote Sensing Letters, pp. $1-5$.

Li, G., Li, Y., \& Han, B., 2017. Accurate interpolation at receiver positions: A novel method for frequency-domain marine CSEM finite-difference modelling, Pure and Applied Geophysics, 174(5), 
2143-2160.

Li, Y. \& Li, G., 2016. Electromagnetic field expressions in the wavenumber domain from both the horizontal and vertical electric dipoles, Journal of Geophysics and Engineering, 13(4), 505-515.

Mackie, R. L., Madden, T. R., \& Wannamaker, P. E., 1993. Three-dimensional magnetotelluric modeling using difference equations - theory and comparisons to integral equation solutions, Geophysics, $\mathbf{5 8}(2), 215-226$.

Martin, R., Komatitsch, D., \& Ezziani, A., 2009. An unsplit convolutional perfectly matched layer technique improved at grazing incidence for the viscoelastic wave equation, Geophysics, 179(1), $333-344$

Mittet, R., 2010. High-order finite-difference simulations of marine CSEM surveys using a correspondence principle for wave and diffusion fields, Geophysics, 75(1), F33-F50.

Newman, G. A. \& Alumbaugh, D. L., 1995. Frequency-domain modelling of airborne electromagnetic responses using staggered finite differences1, Geophysical Prospecting, 43(8), 1021-1042.

Oldenburg, D. W., Haber, E., \& Shekhtman, R., 2013. Three dimensional inversion of multisource time domain electromagnetic data, Geophysics, 78(1), E47-E57.

Pan, G., Abubakar, A., \& Habashy, T. M., 2012. An effective perfectly matched layer design for acoustic fourth-order frequency-domain finite-difference scheme, Geophysical Journal International, 188, 211-222.

Roden, J. A. \& Gedney, S. D., 2000. Convolutional PML (CPML): An efficient FDTD implementation of the CFS-PML for arbitrary media, Microwave and optical technology letters, 27(5), 334-338.

Sasaki, Y. \& Meju, M. A., 2009. Useful characteristics of shallow and deep marine CSEM responses inferred from 3D finite-difference modeling, Geophysics, 74(5), F67-F76.

Schwarzbach, C., Brner, R.-U., \& Spitzer, K., 2011. Three-dimensional adaptive higher order finite element simulation for geo-electromagnetics-a marine CSEM example, Geophysical Journal International, 187(1), 63-74.

Smith, J. T., 1996. Conservative modeling of 3-D electromagnetic fields, part I: Properties and error analysis, Geophysics, 61(5), 1308-1318.

Streich, R., 2009. 3D finite-difference frequency-domain modeling of controlled-source electromagnetic data: Direct solution and optimization for high accuracy, Geophysics, 74(5), F95-F105.

Streich, R., Becken, M., \& Ritter, O., 2013. Robust processing of noisy land-based controlled-source electromagnetic data, Geophysics, 78(5), E237-E247.

Weiss, C. J. \& Constable, S., 2006. Mapping thin resistors and hydrocarbons with marine EM methods, part II - modeling and analysis in 3D, Geophysics, 71(6), G321-G332.

Yee, K., 1966. Numerical solution of initial boundary value problems involving Maxwell's equations in isotropic media, IEEE Transactions on Antennas and Propagation, 14(3), 302-307.

Zhang, Y., 2017. Parallel goal-oriented adaptive finite element modeling for 3D electromagnetic exploration, Ph.D. thesis, University of California, San Diego. 


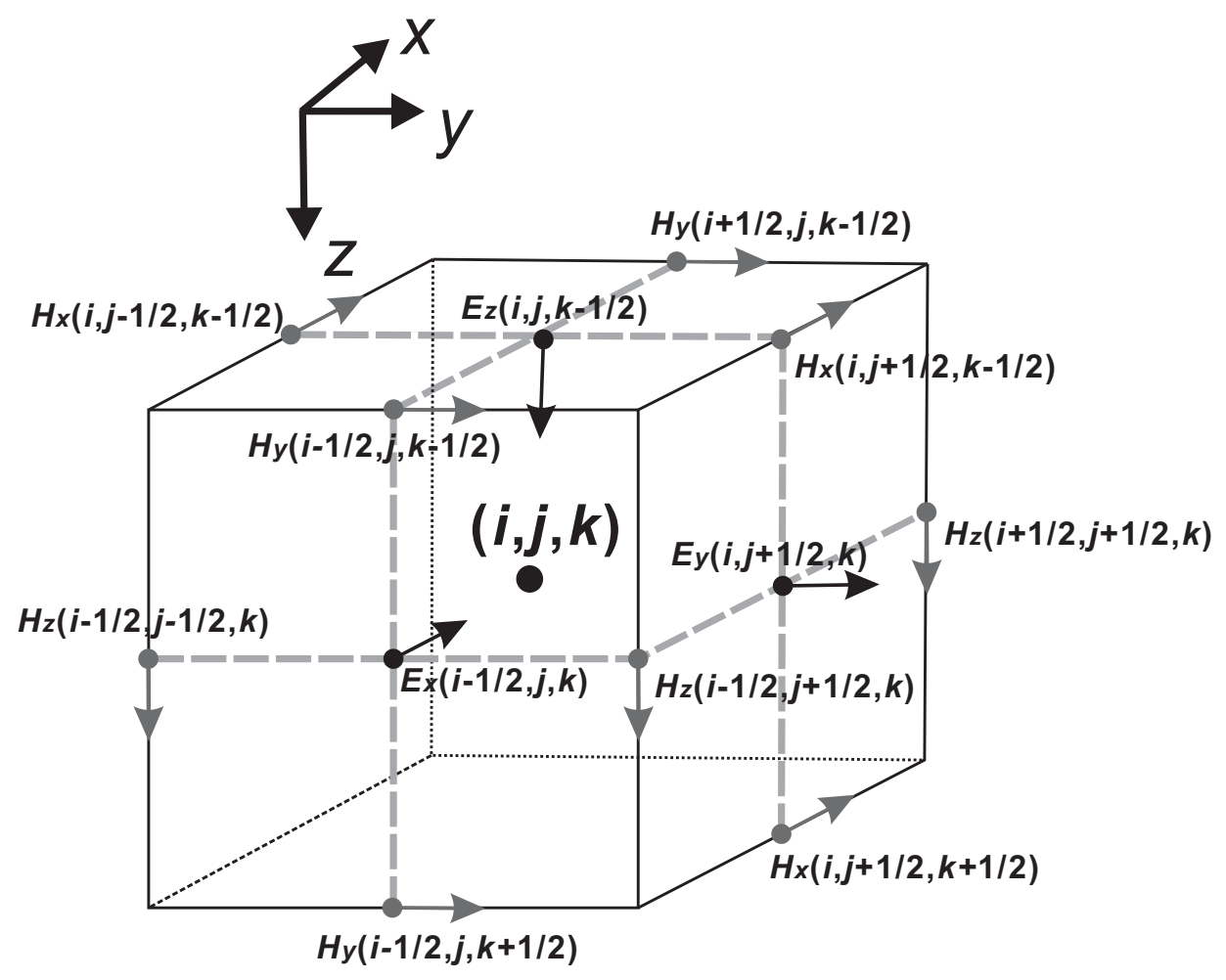

Figure A1. The 3D staggered-grid for cell centered at $(i, j, k)$, where $\Delta x(i), \Delta y(j)$ or $\Delta z(k)$ is the cell size along $x$-, $y$ - and $z$-direction, respectively. The electric field components $E_{x}, E_{y}$ and $E_{z}$ are sampled at the center of cell surfaces, while the magnetic field components $H_{x}, H_{y}$ and $H_{z}$ are sampled at the midpoint of cell edges.

Zhdanov, M. S., 2010. Electromagnetic geophysics: Notes from the past and the road ahead, Geophysics, 75(5), 75A49-75A66.

\section{APPENDIX A: THE STAGGERED FINITE-DIFFERENCE (SFD) DISCRETIZATION}

We use the staggered gridding discretization for the governing equation (3). The staggered finite-difference discretization around cell $(i, j, k)$ is shown in Fig. A1. For the root grid node $(i, j, k)$ located in the cell centers, the electric field components $E_{x}, E_{y}$, and $E_{z}$ are sampled on the cell faces while the magnetic fields $H_{x}, H_{y}$, and $H_{z}$ are on the edges.

From equation (3), we obtain

$$
\begin{aligned}
& \frac{1}{\gamma_{y}^{h}} \frac{\partial}{\partial y}\left(\frac{1}{\gamma_{x}^{e}} \frac{\partial E_{y}^{S}}{\partial x}-\frac{1}{\gamma_{y}^{e}} \frac{\partial E_{x}^{S}}{\partial y}\right)+\frac{1}{\gamma_{z}^{h}} \frac{\partial}{\partial z}\left(\frac{1}{\gamma_{x}^{e}} \frac{\partial E_{z}^{S}}{\partial x}-\frac{1}{\gamma_{z}^{e}} \frac{\partial E_{x}^{S}}{\partial z}\right) \\
& -i \omega \mu_{0} \sigma^{*} E_{x}^{S}=i \omega \mu_{0}\left(\sigma^{*}-\sigma^{P *}\right) E_{x}^{P}, \\
& \frac{1}{\gamma_{z}^{h}} \frac{\partial}{\partial z}\left(\frac{1}{\gamma_{y}^{e}} \frac{\partial E_{z}^{S}}{\partial y}-\frac{1}{\gamma_{z}^{e}} \frac{\partial E_{y}^{S}}{\partial z}\right)+\frac{1}{\gamma_{x}^{h}} \frac{\partial}{\partial x}\left(\frac{1}{\gamma_{y}^{e}} \frac{\partial E_{x}^{S}}{\partial y}-\frac{1}{\gamma_{x}^{e}} \frac{\partial E_{y}^{S}}{\partial x}\right)
\end{aligned}
$$




$$
\begin{aligned}
& -i \omega \mu_{0} \sigma^{*} E_{y}^{S}=i \omega \mu_{0}\left(\sigma^{*}-\sigma^{P *}\right) E_{y}^{P}, \\
& \frac{1}{\gamma_{x}^{h}} \frac{\partial}{\partial x}\left(\frac{1}{\gamma_{z}^{e}} \frac{\partial E_{x}^{S}}{\partial z}-\frac{1}{\gamma_{x}^{e}} \frac{\partial E_{z}^{S}}{\partial x}\right)+\frac{1}{\gamma_{y}^{h}} \frac{\partial}{\partial y}\left(\frac{1}{\gamma_{z}^{e}} \frac{\partial E_{y}^{S}}{\partial z}-\frac{1}{\gamma_{y}^{e}} \frac{\partial E_{z}^{S}}{\partial y}\right) \\
& -i \omega \mu_{0} \sigma^{*} E_{z}^{S}=i \omega \mu_{0}\left(\sigma^{*}-\sigma^{P *}\right) E_{z}^{P},
\end{aligned}
$$

where $\gamma_{\nu}^{e}$ and $\gamma_{\nu}^{h}(\nu=x, y, z)$ are the complex stretched coordinate factors for electric and magnetic field components, respectively.

Following Newman \& Alumbaugh (1995) and Streich (2009), the staggered grid FD discretization for the $x-, y$-, and $z$ - components of the electric field evaluated at $\left(i+\frac{1}{2}, j, k\right)$, $\left(i, j+\frac{1}{2}, k\right)$, and $\left(i, j, k+\frac{1}{2}\right)$ can be written, respectively, as

$$
\begin{aligned}
& \frac{1}{\gamma_{j}^{y h}} \frac{1}{\Delta y_{j}}\left[\frac{E_{i+1, j+\frac{1}{2}, k}^{y S}-E_{i, j+\frac{1}{2}, k}^{y S}}{\gamma_{i+\frac{1}{2}}^{x e} \Delta x_{i+\frac{1}{2}}}-\frac{E_{i+1, j-\frac{1}{2}, k}^{y S}-E_{i, j-\frac{1}{2}, k}^{y^{S}}}{\gamma_{i+\frac{1}{2}}^{x e} \Delta x_{i+\frac{1}{2}}}\right] \\
& -\frac{1}{\gamma_{j}^{y h}} \frac{1}{\Delta y_{j}}\left[\frac{E_{i+\frac{1}{2}, j+1, k}^{x S}-E_{i+\frac{1}{2}, j, k}^{x S}}{\gamma_{j+\frac{1}{2}}^{y e} \Delta y_{j+\frac{1}{2}}}-\frac{E_{i+\frac{1}{2}, j, k}^{x S}-E_{i+\frac{1}{2}, j-1, k}^{x^{S}}}{\gamma_{j-\frac{1}{2}}^{y e} \Delta y_{j-\frac{1}{2}}}\right] \\
& +\frac{1}{\gamma_{k}^{z h}} \frac{1}{\Delta z_{k}}\left[\frac{E_{i+1, j, k+\frac{1}{2}}^{z S}-E_{i, j, k+\frac{1}{2}}^{z S}}{\gamma_{i+\frac{1}{2}}^{x e} \Delta x_{i+\frac{1}{2}}}-\frac{E_{i+1, j, k-\frac{1}{2}}^{z S}-E_{i, j, k-\frac{1}{2}}^{z S}}{\gamma_{i+\frac{1}{2}}^{x e} \Delta x_{i+\frac{1}{2}}}\right] \\
& -\frac{1}{\gamma_{k}^{z h}} \frac{1}{\Delta z_{k}}\left[\frac{E_{i+\frac{1}{2}, j, k+1}^{x S}-E_{i+\frac{1}{2}, j, k}^{x^{S}}}{\gamma_{k+\frac{1}{2}}^{z e} \Delta z_{k+\frac{1}{2}}}-\frac{E_{i+\frac{1}{2}, j, k}^{x S}-E_{i+\frac{1}{2}, j, k-1}^{x S}}{\gamma_{k-\frac{1}{2}}^{z e} \Delta z_{k-\frac{1}{2}}}\right] \\
& -i \omega \mu_{0} \sigma_{i+\frac{1}{2}, j, k}^{*} E_{i+\frac{1}{2}, j, k}^{x S}=i \omega \mu_{0}\left(\sigma_{i+\frac{1}{2}, j, k}^{*}-\sigma_{i+\frac{1}{2}, j, k}^{P *}\right) E_{i+\frac{1}{2}, j, k}^{x P}, \\
& \frac{1}{\gamma_{k}^{z h}} \frac{1}{\Delta z_{k}}\left[\frac{E_{i, j+1, k+\frac{1}{2}}^{z S}-E_{i, j, k+\frac{1}{2}}^{z S}}{\gamma_{j+\frac{1}{2}}^{y e} \Delta y_{j+\frac{1}{2}}}-\frac{E_{i, j+1, k-\frac{1}{2}}^{z S}-E_{i, j, k-\frac{1}{2}}^{z S}}{\gamma_{j+\frac{1}{2}}^{y e} \Delta y_{j+\frac{1}{2}}}\right] \\
& -\frac{1}{\gamma_{k}^{z h}} \frac{1}{\Delta z_{k}}\left[\frac{E_{i, j+\frac{1}{2}, k+1}^{y S}-E_{i, j+\frac{1}{2}, k}^{y S}}{\gamma_{k+\frac{1}{2}}^{z e} \Delta z_{k+\frac{1}{2}}}-\frac{E_{i, j+\frac{1}{2}, k}^{y S}-E_{i, j+\frac{1}{2}, k-1}^{y S}}{\gamma_{k-\frac{1}{2}}^{z e} \Delta z_{k-\frac{1}{2}}}\right] \\
& +\frac{1}{\gamma_{i}^{x h}} \frac{1}{\Delta x_{i}}\left[\frac{E_{i+\frac{1}{2}, j+1, k}^{x S}-E_{i+\frac{1}{2}, j, k}^{x S}}{\gamma_{j+\frac{1}{2}}^{y e} \Delta y_{j+\frac{1}{2}}}-\frac{E_{i-\frac{1}{2}, j+1, k}^{x S}-E_{i-\frac{1}{2}, j, k}^{x S}}{\gamma_{j+\frac{1}{2}}^{y e} \Delta y_{j+\frac{1}{2}}}\right] \\
& -\frac{1}{\gamma_{i}^{x h}} \frac{1}{\Delta x_{i}}\left[\frac{E_{i+1, j+\frac{1}{2}, k}^{y S}-E_{i, j+\frac{1}{2}, k}^{y S}}{\gamma_{i+\frac{1}{2}}^{x e} \Delta x_{i+\frac{1}{2}}}-\frac{E_{i, j+\frac{1}{2}, k}^{y S}-E_{i-1, j+\frac{1}{2}, k}^{y S}}{\gamma_{i-\frac{1}{2}}^{x e} \Delta x_{i-\frac{1}{2}}}\right] \\
& -\quad i \omega \mu_{0} \sigma_{i, j+\frac{1}{2}, k}^{*} E_{i, j+\frac{1}{2}, k}^{y S}=i \omega \mu_{0}\left(\sigma_{i, j+\frac{1}{2}, k}^{*}-\sigma_{i, j+\frac{1}{2}, k}^{P *}\right) E_{i, j+\frac{1}{2}, k}^{y P}, \\
& \frac{1}{\gamma_{i}^{x h}} \frac{1}{\Delta x_{i}}\left[\frac{E_{i+\frac{1}{2}, j, k+1}^{x S}-E_{i+\frac{1}{2}, j, k}^{x S}}{\gamma_{k+\frac{1}{2}}^{z e} \Delta z_{k+\frac{1}{2}}}-\frac{E_{i-\frac{1}{2}, j, k+1}^{x S}-E_{i-\frac{1}{2}, j, k}^{x S}}{\gamma_{k+\frac{1}{2}}^{z e} \Delta z_{k+\frac{1}{2}}}\right]
\end{aligned}
$$




$$
\begin{aligned}
& -\frac{1}{\gamma_{i}^{x h}} \frac{1}{\Delta x_{i}}\left[\frac{E_{i+1, j, k+\frac{1}{2}}^{z S}-E_{i, j, k+\frac{1}{2}}^{z S}}{\gamma_{i+\frac{1}{2}}^{x e} \Delta x_{i+\frac{1}{2}}}-\frac{E_{i, j, k+\frac{1}{2}}^{z S}-E_{i-1, j, k+\frac{1}{2}}^{z S}}{\gamma_{i-\frac{1}{2}}^{x e} \Delta x_{i-\frac{1}{2}}}\right] \\
& +\frac{1}{\gamma_{j}^{y h}} \frac{1}{\Delta y_{j}}\left[\frac{E_{i, j+\frac{1}{2}, k+1}^{y S}-E_{i, j+\frac{1}{2}, k}^{y S}}{\gamma_{k+\frac{1}{2}}^{z e} \Delta z_{k+\frac{1}{2}}}-\frac{E_{i, j-\frac{1}{2}, k+1}^{y S}-E_{i, j-\frac{1}{2}, k}^{y S}}{\gamma_{k+\frac{1}{2}}^{z e} \Delta z_{k+\frac{1}{2}}}\right] \\
& -\frac{1}{\gamma_{j}^{y h}} \frac{1}{\Delta y_{j}}\left[\frac{E_{i, j+1, k+\frac{1}{2}}^{z S}-E_{i, j, k+\frac{1}{2}}^{z S}}{\gamma_{j+\frac{1}{2}}^{y e} \Delta y_{j+\frac{1}{2}}}-\frac{E_{i, j, k+\frac{1}{2}}^{z S}-E_{i, j-1, k+\frac{1}{2}}^{z S}}{\gamma_{j-\frac{1}{2}}^{y e} \Delta y_{j-\frac{1}{2}}}\right] \\
& -i \omega \mu_{0} \sigma_{i, j, k+\frac{1}{2}}^{*} E_{i, j, k+\frac{1}{2}}^{z S}=i \omega \mu_{0}\left(\sigma_{i, j, k+\frac{1}{2}}^{*}-\sigma_{i, j, k+\frac{1}{2}}^{P *} E_{i, j, k+\frac{1}{2}}^{z P},\right.
\end{aligned}
$$

where $\Delta x_{i+\frac{1}{2}}, \Delta y_{j+\frac{1}{2}}$ and $\Delta z_{k+\frac{1}{2}}$ are the midpoint distances between $\Delta x_{i}$ and $\Delta x_{i+1}, \Delta y_{j}$ and $\Delta y_{j+1}$, and $\Delta z_{k}$ and $\Delta z_{k+1}$, respectively.

We can obtain an asymmetric system matrix (not Hermitian) equal to that resulting from a finite volume approach given by Weiss \& Constable (2006) by multiplying the corresponding cell volume, i.e., eq. (A.4) by $\left(\gamma_{i+\frac{1}{2}}^{x e} \Delta x_{i+\frac{1}{2}}\right)\left(\gamma_{j}^{y h} \Delta y_{j}\right)\left(\gamma_{k}^{z h} \Delta z_{k}\right)$, eq. (A.5) by $\left(\gamma_{i}^{x h} \Delta x_{i}\right)\left(\gamma_{j+\frac{1}{2}}^{y e} \Delta y_{j+\frac{1}{2}}\right)\left(\gamma_{k}^{z h} \Delta z_{k}\right)$, and eq. (A.6) by $\left(\gamma_{i}^{x h} \Delta x_{i}\right)\left(\gamma_{j}^{y h} \Delta y_{j}\right)\left(\gamma_{k+\frac{1}{2}}^{z e} \Delta z_{k+\frac{1}{2}}\right)$.

We assume model conductivity and permittivity as constant within each cell. As the electric field components are sampled at cell faces where the complex conductivity could be discontinuous, we employ a harmonic averaging method introduced by Smith (1996). For example, the $x$-, $y$ - and $z$ - staggered complex conductivity is

$$
\begin{aligned}
\sigma_{i+\frac{1}{2}, j, k}^{*} & =\frac{2 \Delta x_{i+\frac{1}{2}}}{\Delta x_{i} / \sigma_{i, j, k}^{*}+\Delta x_{i+1} / \sigma_{i+1, j, k}^{*}}, \\
\sigma_{i, j+\frac{1}{2}, k}^{*} & =\frac{2 \Delta y_{j+\frac{1}{2}}}{\Delta y_{j} / \sigma_{i, j, k}^{*}+\Delta y_{j+1} / \sigma_{i, j+1, k}^{*}}, \\
\sigma_{i, j, k+\frac{1}{2}}^{*} & =\frac{2 \Delta z_{k+\frac{1}{2}}}{\Delta z_{k} / \sigma_{i, j, k}^{*}+\Delta z_{k+1} / \sigma_{i, j, k+1}^{*}},
\end{aligned}
$$

where $\Delta x_{i+\frac{1}{2}}$ is the width of the staggered grid cell that extends between the centers of cell $i$ and $i+1$. The harmonic averaging ensures that the current density derived from nonaveraged and averaged conductivities cross cell boundaries continuous (Smith 1996; Haber et al. 2000; Streich 2009; Li et al. 2016). The harmonic averaging ensures that the current density derived from nonaveraged and averaged conductivities cross cell boundaries continuous. For example, the $x$-, $y$ - and $z$ - staggered complex conductivities are expressed as

$$
\begin{aligned}
\sigma_{i, j, k}^{*} E_{i, j, k}^{x} & =\sigma_{i+1, j, k}^{*} E_{i+1, j, k}^{x} \\
& =\sigma_{i+\frac{1}{2}, j, k}^{*} \frac{\Delta x_{i} E_{i, j, k}^{x}+\Delta x_{i+1} E_{i+1, j, k}^{x}}{\Delta x_{i}+\Delta x_{i+1}} \\
\sigma_{i, j, k}^{*} E_{i, j, k}^{y} & =\sigma_{i, j+1, k}^{*} E_{i, j+1, k}^{y}
\end{aligned}
$$




$$
\begin{aligned}
& =\sigma_{i, j+\frac{1}{2}, k}^{*} \frac{\Delta y_{j} E_{i, j, k}^{y}+\Delta y_{j+1} E_{i, j+1, k}^{y}}{\Delta y_{j}+\Delta y_{j+1}}, \\
\sigma_{i, j, k}^{*} E_{i, j, k}^{z} & =\sigma_{i, j, k+1}^{*} E_{i, j, k+1}^{z} \\
& =\sigma_{i, j, k+\frac{1}{2}}^{*} \frac{\Delta z_{k} E_{i, j, k}^{z}+\Delta z_{k+1} E_{i, j, k+1}^{z}}{\Delta z_{k}+\Delta z_{k+1}} .
\end{aligned}
$$

